\title{
DISSECTING THE REGION OF 3EG J1837-0423 AND HESS J1841-055 WITH INTEGRAL
}

\author{
V. Sguera ${ }^{1}$, G. E. Romero ${ }^{2,3}$, A. Bazzano ${ }^{4}$, N. Masetti ${ }^{1}$, A. J. Bird ${ }^{5}$, and L. Bassani ${ }^{1}$ \\ ${ }^{1}$ IASF/INAF Bologna, via Piero Gobetti 101, 40129 Bologna, Italy \\ 2 Instituo Argentino de Radioastronomia, CCT La Plata-CONICET, C.C.5, (1894) Villa Elisa, Buenos Aires, Argentina \\ ${ }^{3}$ Facultad de Cs. Astronomicas y Geofisicas, UNLP, Paseo del Bosque S/N, (1900) La Plata, Argentina \\ ${ }^{4}$ IASF/INAF Roma, via Fosso del Cavaliere 100, 00133 Roma, Italy \\ ${ }^{5}$ School of Physics and Astronomy, University of Southampton, Southampton SO17 1BJ, UK \\ Received 2008 August 5; accepted 2009 March 12; published 2009 May 11
}

\begin{abstract}
3EG J1837-0423 and HESS J1841-055 are two unidentified and peculiar high-energy sources located in the same region of the sky, separated by $\sim 1.4 \mathrm{deg}$. Specifically, 3EG J1837-0423 is a transient MeV object detected by EGRET only once during flaring activity that lasted a few days while HESS J1841-055 is a highly extended $\mathrm{TeV}$ source. We attempted to match the high-energy emission from the unidentified sources 3EG J18370423 and HESS J1841-055 with X-rays (4-20 keV) and soft $\gamma$-rays (20-100 keV) candidate counterparts detected through deep International Gamma-Ray Astrophysics Laboratory observations of the sky region. As a result we propose the Supergiant Fast X-ray Transient (SFXT) AX J1841.0-0536 as a possible candidate counterpart of 3EG J1837-0423, based on spatial proximity and transient behavior. Alternatively, AX J1841.00536 could be responsible for at least a fraction of the entire TeV emission from the extended source HESS J1841-055, based on a striking spatial correlation. In either case, the proposed association is also supported from an energetic standpoint by a theoretical scenario where AX J1841.0-0536 is a low magnetized pulsar which, due to accretion of massive clumps from the supergiant companion donor star, undergoes sporadic changes to transient Atoll-states where a magnetic tower can produce transient jets and as a consequence high-energy emission. In either case (by association with 3EG J1837-0423 or alternatively with HESS J1841055), AX J1841.0-0536 might be the prototype of a new class of Galactic transient $\mathrm{MeV} / \mathrm{TeV}$ emitters.
\end{abstract}

Key words: gamma rays: observations - gamma rays: theory - X-rays: binaries

Online-only material: color figures

\section{INTRODUCTION}

Soft gamma-ray astronomy is a relatively young research field which experienced a golden age in the last decades. Breakthroughs have been achieved thanks to $\gamma$-ray satellites carrying instruments such as Compton Gamma-Ray Observatory (CGRO)/EGRET, International Gamma-Ray Astrophysics Laboratory (INTEGRAL)/IBIS, and Swift/BAT whose survey capabilities unveiled the extreme richness of objects in the soft $\gamma$-ray sky. Recently, ground-based very high energy (VHE) $\gamma$ ray astronomy has also shown rapid progress with important results reported by the third generation of imaging atmospheric Cherenkov telescopes such as HESS, MAGIC, VERITAS, and CANGAROO. A rapidly growing list of $\sim 50$ firmly identified sources have been detected at $\mathrm{TeV}$ energies, including active galactic nuclei (AGNs; 15), pulsar wind nebulae (PWNs; $\sim 18$ ), supernova remnants (SNR; $\sim 10$ ), and X-ray binaries $(\sim 4)$. In addition, there are $\sim 24 \mathrm{TeV}$ sources still unidentified with no firm counterpart at other wavelengths.

Among the different types of $\mathrm{TeV}$ sources, $\gamma$-ray binaries are rapidly becoming a subject of topical and major interest in soft gamma-ray astronomy. The four firm TeV binaries detected so far are systems hosting a bright massive OB star as companion of the compact object, namely, LS 5039 (Aharonian et al. 2005a), LS I+61 303 (Albert et al. 2006), PSR B1259-63 (Aharonian et al. 2005b), and Cygnus X-1 (Albert et al. 2007). Their VHE emission provides evidence that particles can be efficiently accelerated to energies as high as $\sim 30 \mathrm{TeV}$. Different scenarios have been proposed in the last few years to explain the emission mechanism at such high energies from high-mass X-ray binaries (HMXBs). Some are based on the microquasar accretion/jet framework, with both leptonic and hadronic scenarios. In the former, the $\gamma$-ray emission is due to inverse Compton scattering between relativistic electrons in the jet and seed stellar and/ or synchrotron photons (Bosch-Ramon et al. 2006; Paredes et al. 2006; Dermer \& Boettcher 2006) whereas in the latter the radiation originates from inelastic proton-proton interactions between relativistic hadrons from the jet and cold protons or nuclei from the stellar wind (Romero et al. 2003, 2005). Protonphoton interactions are also an interesting possibility for both low- and high-mass microquasars (see Romero \& Vila 2008). An alternative leptonic scenario for the origin of $\gamma$-rays takes into account the region of interaction between the relativistic wind of a young neutron star and the wind of the stellar companion (Maraschi \& Treves 1981; Tavani \& Arons 1997; Dubus 2006; see Romero et al. 2007a for a comparison between the pulsar wind and microquasar models). Finally, variable hadronic $\gamma$ ray emission could be produced in transient HMXBs through the Cheng-Ruderman mechanism in the magnetosphere of an accreting neutron star (Orellana et al. 2007a).

In this paper, we report on new INTEGRAL imaging data of the sky region containing the two still unidentified $\gamma$-ray sources 3EG J1837-0423 and HESS J1841-055. Our goal is to find their best candidate counterparts, to this aim we discuss their spatial and temporal relationship with the nearby sources detected by INTEGRAL in X-rays (4-20 keV) and soft $\gamma$-rays (20-100 keV).

\section{THE MeV/TeV EMITTING REGION}

3EG J1837-0423 and HESS J1841-055 are two unidentified and peculiar high-energy sources located in the same 


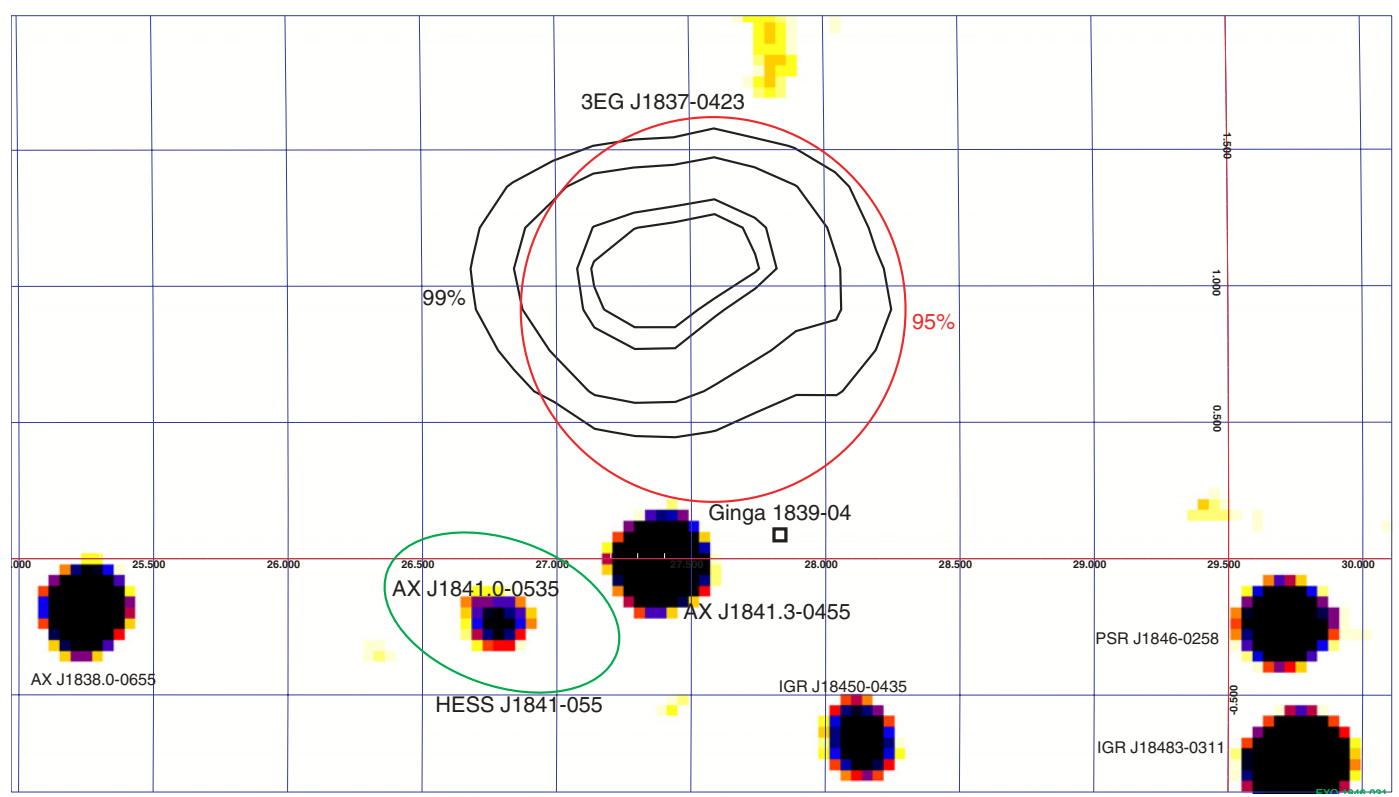

Figure 1. IBIS/ISGRI mosaic significance map (20-100 keV, $\sim 3 \mathrm{Ms}$ exposure time) of the region including the two unidentified high-energy sources HESS J1841-055 (ellipse) and 3EG J1837-0423 (50\%, 68\%, 95\%, and 99\% probability contours as taken from the 3EG catalog and 95\% error circle as taken from the revised 3EG catalog). Also shown is the position of the X-ray source GS 1839-04 (square), not detected in the mosaic.

(A color version of this figure is available in the online journal.)

region of the sky. Their angular separation is $\sim 1.4 \mathrm{deg}$ (see Figure 1).

HESS J1841-055 is an unidentified TeV source discovered by HESS during the Galactic plane survey (Aharonian et al. $2008)$. It shows a highly extended $\left(\sim 24^{\prime}\right.$ semimajor axis) and possibly bipolar morphology in its $\mathrm{TeV}$ image. The spectrum is best fitted by a power law with photon index $\Gamma \sim 2.4$, the flux is $\sim 5.8 \times 10^{-11} \mathrm{erg} \mathrm{cm}^{-2} \mathrm{~s}^{-1}(0.5-80 \mathrm{TeV})$ with a corresponding luminosity of $\sim 3.3 \times 10^{35} \mathrm{erg} \mathrm{s}^{-1}$, assuming a distance of $\sim 6.9$ $\mathrm{kpc}$ (see Section 4.3.2). The extended TeV morphology suggests that HESS J1841-055 is the blend of more than one source and to date no obvious unique counterpart has been found at other wavelengths for the entire source. Aharonian et al. (2008) searched for possible counterparts using standard catalogs of sources thought to be responsible of VHE emission, i.e., PWNs, SNRs, HMXBs. By doing so, they found four candidates each of which could be responsible for at least part of the entire $\mathrm{TeV}$ emission: the two pulsars PSR J1841-0524 and PSR J18380549, the diffuse source G26.6-0.1 which is a candidate SNR based on its ASCA spectrum and finally the HMXB AX J1841.00536.

3EG J1837-0423 is an unidentified EGRET source $(E>100$ $\mathrm{MeV}$ ) listed in the third EGRET catalog 3EG (Hartman et al. $1999)$ with coordinates and $95 \%$ probability contour radius equal to R.A. $=279.4 \mathrm{deg}$, decl. $=-4.4 \mathrm{deg}$ and $\sim 0.5 \mathrm{deg}$, respectively. Recently Casandjian \& Grenier (2008) reported a revised version of the 3EG catalog based on the reanalysis of the whole EGRET data set by using a new and much improved galactic interstellar emission model based on very recent dark gas, CO, H I, and interstellar radiation field data. 3EG J18370423 is listed in such revised version of the 3EG catalog with a bigger $95 \%$ probability contour radius $(\sim 0.7 \mathrm{deg})$ and very similar coordinates $($ R.A. $=279.6 \mathrm{deg}$, decl. $=-4.34 \mathrm{deg}$ ) with respect to those reported in the 3EG catalog. 3EG J1837-0423 is a very peculiar transient source discovered in 1995 (viewing period June 20-30) during a very bright $\gamma$-ray flare lasting only a few days (Tavani et al. 1997) and reaching a peak flux above
$100 \mathrm{MeV}$ of $\sim 6.4 \times 10^{-10} \mathrm{erg} \mathrm{cm}^{-2} \mathrm{~s}^{-1}$. The corresponding $\gamma$ ray luminosity is $\sim 3.6 \times 10^{36} \mathrm{erg} \mathrm{s}^{-1}$, assuming again a distance of $\sim 6.9 \mathrm{kpc}$ (see Section 4.3.2). When active, 3EG J1837-0423 was the second brightest $\gamma$-ray source in the sky. The photon spectrum above $30 \mathrm{MeV}$ during the peak emission is best fit by a power law with spectral index equal to $\sim 2.1$. EGRET pointed in the direction of 3EG J1837-0423 15 more times during a period of $\sim 4$ years and each observation was typically $1-2$ weeks long; the source was never significantly detected again except during the viewing period 1994 July $18-25$ and only with a marginal significance $(\sim 3 \sigma)$. Such marked transient behavior is strongly reminiscent of a blazar, but the $99 \%$ error circle of 3EG J18370423 contains no radio object at a flux level consistent with other blazars seen by EGRET (Tavani et al. 1997).

As can be clearly seen in Figure 1, HESS J1841-055 and 3EG J1837-0423 are closely located in the sky. At first glance, their considerably large positional uncertainty regions could misleadingly suggest a spatial association and a common nature. However, this is implausible because of their completely different high-energy characteristics (HESS J1841-055 is a highly extended and nonvariable source while 3EG J18370423 is a pointlike and transient source), moreover the chance probability of positional coincidence between all galactic HESS sources and EGRET objects is as high as $\sim 10 \%$ (Funk et al. 2008). Our goal is to find their best candidate counterpart and to this aim we consider HESS J1841-055 and 3EG J1837-0423 as two distinct sources, not physically associated. In the following sections, we report a study of the field containing both highenergy sources, using data obtained with INTEGRAL in the energy bands 4-20 keV and 20-100 keV.

\section{INTEGRAL OBSERVATIONS OF THE $\mathrm{MeV} / \mathrm{TeV}$ EMITTING REGION}

\subsection{Data Analysis}

INTEGRAL data collected with JEM-X (Lund et al. 2003) and IBIS (Ubertini et al. 2003) have been considered in this work. 


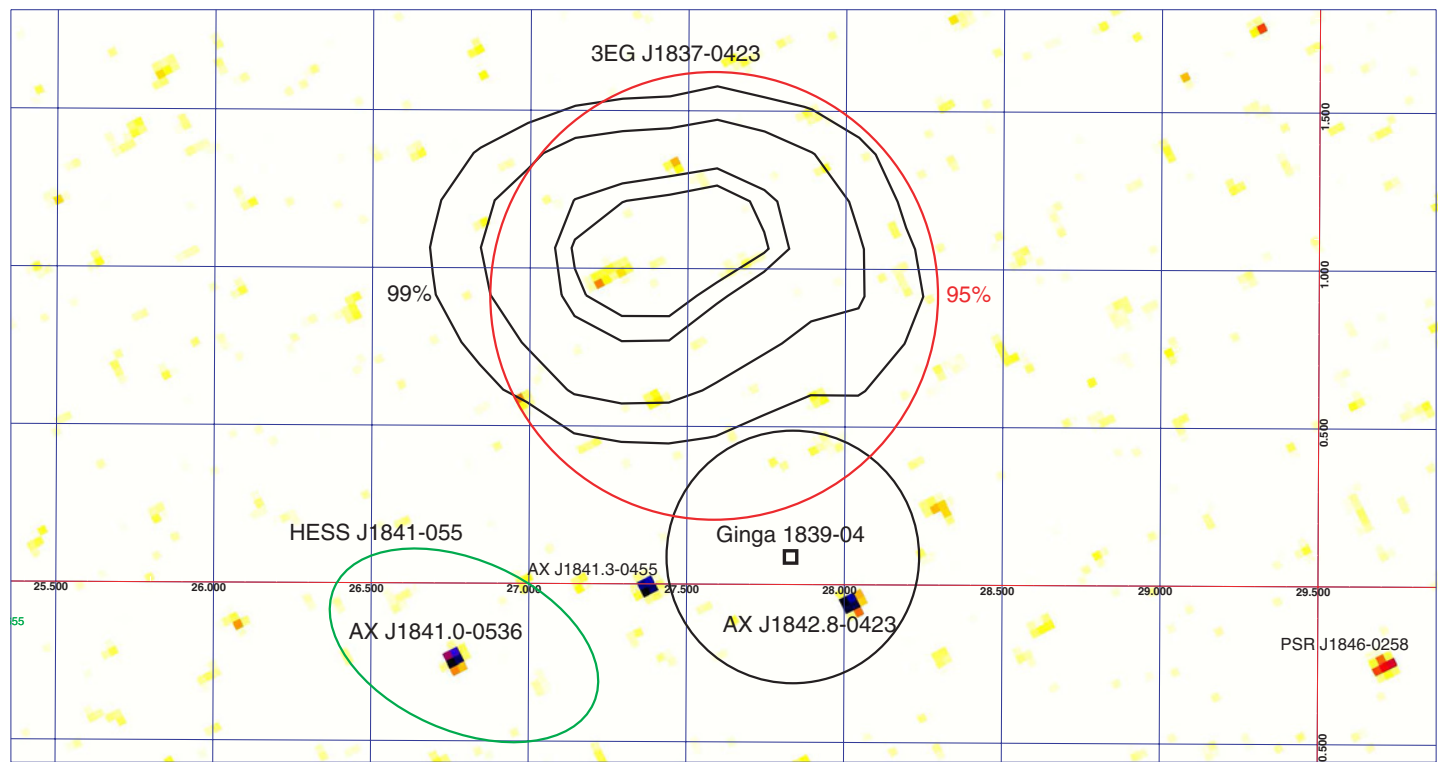

Figure 2. JEM-X1 mosaic significance map (4-20 keV, 130 ks exposure time) of the region including HESS J1841-055 (ellipse) and 3EG J1837-0423 (50\%, 68\%, 95\%, and 99\% probability contours as taken from the 3EG catalog and 95\% error circle (bigger) as taken from the revised 3EG catalog). The smaller circle represents the uncertainty region of GS $1839-04$.

(A color version of this figure is available in the online journal.)

The data reduction was carried out with the release 7.0 of the Offline Scientific Analysis (OSA) software. INTEGRAL observations are typically divided into short pointings called Science Windows (ScWs) of $\sim 2000 \mathrm{~s}$ duration. Through the paper, the spectral analysis was performed using Xspec version 11.3 and all spectral uncertainties are given at the $90 \%$ confidence level for a single parameter of interest.

\subsection{INTEGRAL Imaging}

Because of the regular monitoring of the Galactic plane by INTEGRAL, the sky region including the two unidentified highenergy sources HESS J1841-055 and 3EG J1837-0423 is now well covered by JEM-X (4-20 keV) and IBIS (20-100 keV), with total exposure times of $\sim 160 \mathrm{ks}$ and $\sim 3 \mathrm{Ms}$, respectively.

Figure 1 shows the $20-100 \mathrm{keV}$ IBIS significance mosaic map with superimposed the error region of HESS J1841-055 (ellipse) and 3EG J1837-0423 (50\%-99\% probability contours as taken from the 3EG catalog and $95 \%$ error circle as taken from the revised 3EG catalog). We note that AX J1841.0-0536 is the only soft $\gamma$-ray source to be detected within the HESS error ellipse, the other likely candidates proposed by Aharonian et al. (2008) are not visible in the IBIS map and their estimated $2 \sigma$ upper limits are $\sim 0.2$ mcrab (or $1.5 \times 10^{-12} \mathrm{erg} \mathrm{cm}^{-2}$ $\mathrm{s}^{-1}$ ) and $\sim 0.4 \mathrm{mcrab}$ (or $3.7 \times 10^{-12} \mathrm{erg} \mathrm{cm}^{-2} \mathrm{~s}^{-1}$ ) in the 20-40 and 40-100 keV energy bands, respectively. As for 3EG J1837-0423, no soft $\gamma$-ray sources have been detected inside its uncertainty contours, however, we note that two objects have been detected in its immediate nearness: AX J1841.3-0455 and AX J1841.0-0536.

Moreover, we took into account the possible contribution, inside or in the proximity of EGRET and HESS error regions, from other cataloged soft $\gamma$-ray sources not detected in the IBIS mosaic in Figure 1. To this aim, we used the INTEGRAL reference catalog which classifies previously known X-ray and $\gamma$-ray sources that are, or have been at least once, brighter than $\sim 1$ mcrab above $3 \mathrm{keV}$ and then expected to be detected by INTEGRAL. The cross-correlation with the HESS uncertainty region resulted in only one cataloged object, AX J1841.0-0536, which is visible in Figure 1. Concerning the EGRET uncertainty regions, the cross-correlation resulted in three cataloged objects: AX J1841.0-0536, AX J1841.3-0455 (both visible in Figure 1), and GS $1839-04$ that is not detected above $20 \mathrm{keV}$ but is nevertheless indicated in Figure 1 with a square.

Next, we searched the entire public data archive of JEM-X1 (from revolution 171 to 528) and JEM-X2 (from revolution 46 to 170) for pointings where HESS J1841-055 and 3EG J18370423 were within the fully coded field of view (FOV) of JEM-X $(\sim 2.4 \mathrm{deg})$. As a result, a total of $24 \mathrm{ScWs}(\mathrm{JEM}-\mathrm{X} 2)$ and 105 ScWs (JEM-X1) were selected, spanning the viewing periods from 2003 March 11 to 2003 October 16 and 2004 March 10 to 2006 September 2, respectively. We used all available ScWs to generate a mosaic significance map in the $4-20 \mathrm{keV}$ band with a total exposure of $\sim 130 \mathrm{ks}$ (JEM-X1) and $\sim 30 \mathrm{ks}$ (JEM$\mathrm{X} 2$ ). Figure 2 shows the JEM-X significance map having the longest exposure (JEM-X1) again with the superimposition of the HESS J1841-055 and 3EG J1837-0423 uncertainty regions. Apart from AX J1841.0-0536 and AX J1841.3-0455, there is another JEM-X detection $(\sim 5 \sigma$ level $)$ at R.A. $=184240.8$, decl. $=-042341.9$ (error radius $\sim 3^{\prime}$ ). This position is fully compatible with that of the cataloged X-ray source AX J1842.80423 (R.A. $=1842$ 48, decl. $=-042300$, error radius $\sim 3^{\prime}$ ). Moreover, Figure 2 shows that AX J1842.8-0423 is very likely associated with the INTEGRAL reference catalog source GS 1839-04 (error radius $\sim 24^{\prime}$ ).

\section{DISSECTING THE MeV/TeV EMITTING REGION}

As stated in the previous section, three sources were detected by INTEGRAL during deep observations of the $\mathrm{MeV} / \mathrm{TeV}$ emitting region: AX J1841.0-0536, AX J1842.8-0423, and AX J1841.3-0455. Only one of them, AX J1841.0-0536, is located inside the uncertainty region of HESS J1841-055: the spatial association is striking being right at the center of the $\mathrm{TeV}$ error ellipse. On the contrary, no object has been detected by INTEGRAL inside the $95 \%$ error circle 
of 3EG J1837-0423 ( $~ 0.7$ deg radius). Then, we looked for counterparts in a bigger error area having a radius of $\sim 1.2 \mathrm{deg}$, i.e., almost twice the $95 \%$ error circle radius. We are aware that this is a dangerous approach because of the possibility that unrelated sources could be included; however, as pointed out by Thompson et al. (1995) and Hartman et al. (1999), the position uncertainty regions provided in the 3EG catalog are statistical only: additional systematic position errors due to inaccuracies in the Galactic diffuse radiation model and source confusion are strongly recommended to the users, particularly for those EGRET sources located on the Galactic plane. As example, the corresponding counterparts of three well-known gamma-ray pulsars (3EG J0534+2200, 3EG J0633+1751, and 3EG J0834-4511) are located well outside their 99\% EGRET probability contours (Hartman et al. 1999). Therefore, it is clearly worth searching for counterparts of unidentified EGRET sources even at large distances, i.e., well outside their error boxes. In the specific case of 3EG J1837-0423, we point out that by using a much improved background model (Casandjian $\&$ Grenier 2008) its $95 \%$ error circle radius increased from $\sim 0.5 \mathrm{deg}$ (3EG catalog) to $\sim 0.7 \mathrm{deg}$ (revised 3EG catalog). Inside an error region of $\sim 1.2 \mathrm{deg}$ radius, we note that the position of the three sources AX J1841.0-0536, AX J1842.80423, and AX J1841.3-0455 might be still fully compatible with 3EG J1837-0423 in view of the systematics and they could be considered as potential counterparts. In this section, we report results from archival and new X-ray/soft $\gamma$-ray observations of AX J1842.8-0423, AX J1841.3-0455, and AX J1841.0-0536. Moreover, we discuss their possible physical association with the corresponding spatially associated high-energy source.

\subsection{AX J1842.8-0423 (Possibly Also GS 1839-04)}

AX J1842.8-0423 is an unidentified transient X-ray source discovered on 1996 October during a $\sim 35$ ks observation of $A S C A$ while surveying the Scutum arm region (Terada et al. 1999). It was undetectable in a previous ASCA observation in 1993 and also in a subsequent one on 1997 April, providing an upper limit to the flux and outburst activity duration of $\sim 2 \times 10^{-13} \mathrm{erg} \mathrm{cm}^{-2} \mathrm{~s}^{-1}(2-10 \mathrm{keV})$ and $\sim$ half a year, respectively. When detected, it exhibited a $2-10 \mathrm{keV}$ flux of $\sim 5 \times 10^{-12} \mathrm{erg} \mathrm{cm} \mathrm{cm}^{-2} \mathrm{~s}^{-1}$ with no variability. The ASCA spectrum is well approximated by an absorbed power law with $\Gamma=2.9 \pm 0.4$ and $N_{\mathrm{H}} \sim 5 \times 10^{22} \mathrm{~cm}^{-2}$; moreover the most interesting spectral property is the strong iron line detected at $\sim 6.8 \mathrm{keV}$ with an equivalent width of $\sim 2.4 \mathrm{keV}$. In general, LMXBs occasionally show iron lines while HMXBs sometimes show significant Fe-K lines which do not normally exhibit an equivalent width in excess of $\sim 1 \mathrm{keV}$ (Nagase 1989). The overall X-ray behavior of AX J1842.8-0423 is indeed quite peculiar and intriguing; Terada et al. (1999, 2001) proposed that it may be explained in terms of a close binary involving a magnetized white dwarf viewed from pole-on inclination. In this case, the unusually strong iron line is interpreted as arising from line-photon collimation in the accretion column of the white dwarf, as a result of resonance scattering of line photons. Such possibility is empirically supported by X-ray observations of some magnetic white dwarf binaries classified as AM Herculistype (Ishida et al. 1998; Misaki et al. 1996).

As stated in the end of Section 3.2, the detection in the JEMX1 significance mosaic map (Figure 2) at R.A. = 1842 40.8, decl. $=-042341.9$ (error radius $\sim 3^{\prime}$ ) is fully compatible with the position of AX J1842.8-0423. Unfortunately, insufficient statistics did not allow us to extract a meaningful JEM-X spectrum and light curve. The 4-20 keV flux was estimated as $\sim 3 \times 10^{-12} \mathrm{erg} \mathrm{cm}^{-2} \mathrm{~s}^{-1}$, i.e., similar to that measured by $A S C A$ in 1996. We point out that AX J1842.8-0423 was not detected in the JEM-X2 mosaic, supporting its transient X-ray behavior. Moreover, a deeper inspection of the total JEM-X1 data set (spanning the time interval from 2004 March 10 to 2006 September 2) revealed that the longest interval of detectability of the source was $\sim 5$ months (from 2006 March 18 to 2006 September 2), i.e., a similar duration activity as constrained by $A S C A$. It is also worth noting that AX J1842.8-0423 falls in the much larger error circle ( $\sim 24^{\prime}$ radius) of the Ginga source GS 1839-04 (Figure 2), which is the only cataloged X-ray source present in $\mathrm{a} \sim 15^{\prime}$ radius circle region around AX J1842.8-0423. GS 1839-04 was discovered by Ginga about two decades ago (Koyama et al. 1989, 1990), during an outburst lasting several days; since then no more X-ray detections have been reported in the literature by other X-ray missions. The Ginga X-ray spectrum was fit by an absorbed power law with $\Gamma=1.9 \pm 0.2$ and X-ray flux of $\sim 6 \times 10^{-12} \mathrm{erg} \mathrm{cm}^{-2} \mathrm{~s}^{-1}(2-10 \mathrm{keV})$. The optical counterpart of GS 1839-04 is still unknown, however, the X-ray source is reported in the latest catalog of HMXBs by Liu et al. (2007), mainly because of the possible discovery by Ginga of $\sim 81 \mathrm{~s}$ pulsation, although at $\sim 4.5 \sigma$ level. We think that the HMXB scenario is uncertain but note that the spatial correlation, the transient X-ray behavior and the X-ray spectral shape and flux, all seem to suggest that GS 1839-04 is very likely AX J1842.8-0423.

AX J1842.8-0423 is located far away from the uncertainty region of HESS J1841-055 but it is close to the 95\% probability contour of 3EG J1837-0423. At first glance, the spatial proximity and the transient X-ray behavior would make AX J1842.8-0423 a possible candidate counterpart of the transient 3EG J1837-0423. However, we consider such an association highly unlikely on the basis of the following findings: AX J1842.8-0423 is very likely a magnetized white dwarf binary system; it has been significantly detected at soft X-rays (4-20 keV) but not above $20 \mathrm{keV}$; its transient X-ray activity (timescale of several weeks) is significantly longer than that recorded by EGRET (timescale of few days).

\subsection{AX J1841.3-0455}

AX J1841.3-0455 is an anomalous X-ray pulsar (AXP) located at the center of the small $\left(\sim 4^{\prime}\right.$ diameter $)$ SNR Kes 73. AXPs are rare objects closely concentrated along the Galactic plane (see Kaspi 2007 for a review). Their "anomalous" Xray luminosities $\left(\sim 10^{33}-10^{35} \mathrm{erg} \mathrm{s}^{-1}, 2-10 \mathrm{keV}\right)$ are orders of magnitude too high to be explained by rotational energy release due to spin-down. On the contrary, the so-called magnetar model, based on the decay of very strong magnetic fields $\left(10^{14}\right.$ $\left.10^{15} \mathrm{G}\right)$, is able to explain the observed characteristics of AXPs. Traditionally, AXPs were considered soft X-ray sources (0.5$10 \mathrm{keV})$ with thermal-like spectra $(\mathrm{kT} \sim 0.4-0.7 \mathrm{keV})$ plus a steep power-law component $(\Gamma \sim 3-4)$. Recently, INTEGRAL has changed this traditional view by detecting bright and persistent hard tail emission described by power-law with $\Gamma$ $\sim 1-1.5$ and no sign of a break up to $\sim 150 \mathrm{keV}$ (Kuiper et al. 2004, 2006). However, since no counterparts have been found in the MeV domain by COMPTEL (0.75-30 MeV) and EGRET $(E>100 \mathrm{MeV})$, these spectra are expected to show breaks.

Different types of X-ray flux variability are displayed by AXPs: from moderate flux changes up to a factor of few on timescales of years to intense burst activity lasting milliseconds to few hours (Kaspi 2007). Specifically, AX J1841.3-0455 is 
known to be a stable AXP (Kaspi 2007), no bursting activity has been recorded over $\sim 20$ years of observations with Ginga, $A S C A, R X T E$, and BeppoSAX. We investigated the long-term IBIS light curve (20-100 keV) of the source, spanning a time interval from 2003 March to 2006 April, and we confirm the persistent hard X-ray emission with no sign of flaring activity. Moreover, we performed a spectral analysis of the IBIS spectrum (20-200 keV) which is best fit by a power law with a hard photon index $(\Gamma=1.55 \pm 0.1)$, i.e., very similar to that reported by Kuiper et al. (2004) using RXTE data.

As we can note from the INTEGRAL significance mosaics in Figures 1 and 2, AX J1841.3-0455 has been significantly detected both by IBIS and JEM-X. It is located far away from HESS J1841-055 (well outside its error ellipse) so it should not be considered as its candidate counterpart. On the contrary, it is close to the $95 \%$ error circle of 3EG J1837-0423. However, we can confidently assert that AX J1841.3-0455 is not physically associated with the unidentified EGRET source in the light of their very different X-ray/soft $\gamma$-ray behaviors as well as of the findings on AXPs reported above.

\subsection{AX J1841.0-0536}

\subsubsection{Archival $X$-ray Observations}

AX J1841.0-0536 (also known as IGR J18410-0536) is a $4.7 \mathrm{~s}$ transient X-ray pulsar discovered by ASCA in 1994 and then detected again in 1999; in both cases it showed a fast X-ray flaring activity with flux increasing from $\sim 10^{-12} \mathrm{erg} \mathrm{cm}^{-2} \mathrm{~s}^{-1}$ to $\sim 10^{-10} \mathrm{erg} \mathrm{cm}^{-2} \mathrm{~s}^{-1}(2-10 \mathrm{keV})$ within only $\sim 1 \mathrm{hr}$ (Bamba et al. 2001). Subsequently, no more X-ray flares were reported in the literature until the launch of INTEGRAL which detected three fast hard X-ray flares having a duration of a few hours and a 20-80 keV peak flux of $\sim 10^{-9} \mathrm{erg} \mathrm{cm}^{-2} \mathrm{~s}^{-1}$ (Rodriguez et al. 2004; Sguera et al. 2006).

A $20 \mathrm{ks}$ Chandra pointed observation in 2004 detected the source during a phase of no major flaring activity (Halpern et al. 2004), the flux level was $\sim 4 \times 10^{-12} \mathrm{erg} \mathrm{cm}^{-2} \mathrm{~s}^{-1}(0.5-10$ $\mathrm{keV}$ ) likely representing the quiescent X-ray emission. Since 2007 October, Swift/XRT has been performing a monitoring campaign of AX J1841.0-0536 (Sidoli et al. 2008) and the source is usually detected with a low-level X-ray activity of $\sim 3.5 \times 10^{-12} \mathrm{erg} \mathrm{cm} \mathrm{cm}^{-2} \mathrm{~s}^{-1}(2-10 \mathrm{keV})$. Finally, we performed a cross-correlation of the Chandra error circle of AX J1841.0-0536 with all radio catalogs available in the HEASARC database. This has resulted in no cataloged objects, suggesting that AX J1841.0-0536 is not a radio emitter; however, a deep pointed radio observation is needed to fully confirm such possibility.

\subsubsection{Optical Properties: Reddening and Distance}

Thanks to the Chandra very accurate position, Halpern et al. (2004) identified the optical counterpart of AX J1841.00536 with USNO-A2.0 0825_12601262, a reddened star with weak $\mathrm{H} \alpha$ in emission (Halpern et al. 2004). Recently, it has been classified as a B1 Ib-type supergiant through IR spectroscopy (Nespoli et al. 2008); this allowed the classification of AX J1841.0-0536 as a member of the newly discovered class of Supergiant Fast X-ray transients (Negueruela et al. 2006; Sguera et al. 2005, 2006).

We estimated reddening and distance for this Supergiant Fast X-ray Transient (SFXT), considering its average optical and NIR absolute magnitudes (Halpern et al. 2004) and colors of an early-type B1 I star (Lang 1992; Wegner 1994). We found that the reddening in the optical $V$ band along the source line of sight is $A_{V} \sim 6 \mathrm{mag}$, applying the Milky Way extinction law by Cardelli et al. (1989). This figure, using the empirical formula of Predehl \& Schmitt (1995), translates into a $N_{\mathrm{H}}$ value of $\sim 1.1 \times 10^{22} \mathrm{~cm}^{-2}$ along the line of sight of AX J1841.0 0536 . When compared with the $N_{\mathrm{H}}$ value derived from our X-ray spectral fitting (see Section 4.3.3), this suggests the presence of additional absorbing material in the vicinity of the X-ray source, likely due to the accretion stream flowing onto the compact object in this X-ray system. Using the absolute and the observed $R$ magnitudes for this source (again assuming that the companion is a B1 I supergiant star) and the estimate of the absorption in the optical-NIR bands, we infer a distance to this source of $\sim 6.9 \pm 1.7 \mathrm{kpc}$; this is consistent with the hypothesis that AX J1841.0-0536 lies in the Sagittarius arm tangent of the Galaxy, possibly on the side closer to the Earth, given the relatively low amount of $A_{V}$ compared to the Galactic one along the source line of sight ( $\sim 53 \mathrm{mag}$, according to Schlegel et al. 1998). We point out that our retrieved value for the distance is compatible, within the uncertainties, with that reported by Nespoli et al. (2008).

\subsubsection{New JEM-X and IBIS/ISGRI Results}

We searched the entire public IBIS data set (from end of 2003 February to end of 2006 September) for new outburst activity from AX J1841.0-0536. Figure 3 shows its long-term 20-60 keV IBIS light curve on the ScW timescale with the flux extracted from each pointing where the source was within $12 \mathrm{deg}$ of the center of the FOV. The black line represents the $2 \sigma$ upper limit at the $\mathrm{ScW}$ level $(\sim 10$ mcrab or $1.2 \times$ $10^{-10} \mathrm{erg} \mathrm{cm}^{-2} \mathrm{~s}^{-1}$ ) and we note that most of the time AX $\mathrm{J} 1841.0-0536$ is in quiescence showing only rare signs of flaring activity above a flux of $\sim 3 \times 10^{-10} \mathrm{erg} \mathrm{cm}^{-2} \mathrm{~s}^{-1}$ (the dashed line in Figure 3). In fact, above this level four fast X-ray flares are evident in the light curve. The flares labeled as A, B, and $\mathrm{C}$ have already been reported in the literature (Rodriguez et al. 2004; Sguera et al. 2006) with very similar duration (a few hours) and peak flux $\left(\sim 10^{-9} \mathrm{erg} \mathrm{cm}^{-2} \mathrm{~s}^{-1}, 20-80 \mathrm{keV}\right)$. The flare D is newly discovered and Figure 4 displays a zoomed view of its light curve. In spite of the gap in the middle due to visibility constraints, it is evident that the source underwent an outburst longer than usual, with a total duration of $\sim 2.5$ days. Initially the flux was consistent with zero and suddenly it flared up to $\sim 50$ mcrab or $6 \times 10^{-10} \mathrm{erg} \mathrm{cm}^{-2} \mathrm{~s}^{-1}(20-60 \mathrm{keV})$ in just $\sim 2 \mathrm{hr}$, then it dropped again to a very low level. Although the gap prevented a full coverage of the outburst activity, we can reasonably assume that another fast X-ray flare, of which we could see the decay phase, took place during the gap. Unfortunately, the statistical quality of the IBIS/ISGRI data is insufficient to perform a pulsation search during the flaring activity.

The IBIS/ISGRI spectrum of the total outburst activity (22$60 \mathrm{keV})$ is best described by a power law with a steep photon index $\Gamma=3.0 \pm 0.35\left(\chi_{v}^{2}=1.45,13\right.$ degree of freedom (dof)) or alternatively by a thermal Bremsstrahlung model with $\mathrm{kT}$ $=19_{-4}^{+5} \mathrm{keV}\left(\chi_{v}^{2}=1.44,13\right.$ dof $)$. Because of the different JEM-X/ISGRI FOV, we have low energy coverage of the outburst during only four ScWs indicated in Figure 4 by the black dots; their extracted JEM-X spectrum (3-15 keV) was best fit by an absorbed power law with $\Gamma=2.1 \pm 0.6$ and $N_{\mathrm{H}}=14_{-12}^{+18} \times 10^{22} \mathrm{~cm}^{-2}$. We note that the latter exceeds the Galactic absorption along the line of sight $\left(\sim 1.9 \times 10^{22} \mathrm{~cm}^{-2}\right)$. Unfortunately, the $N_{\mathrm{H}}$ could not be well constrained because the 


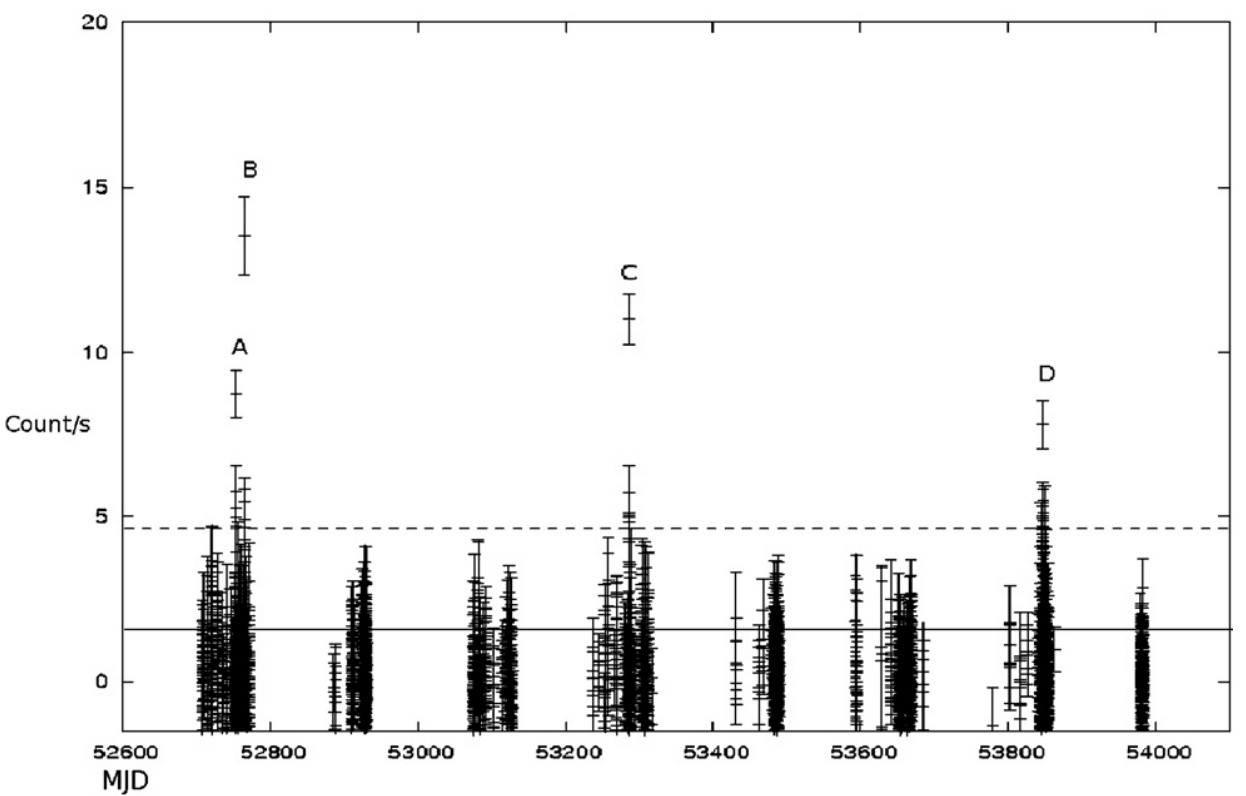

Figure 3. ISGRI long-term light curve (20-60 keV) of AX J1841.0-0536. Time and flux axes are in MJD and count $\mathrm{s}^{-1}$, respectively. Each data point represents the average flux during one $\mathrm{ScW}(\sim 2000 \mathrm{~s})$.

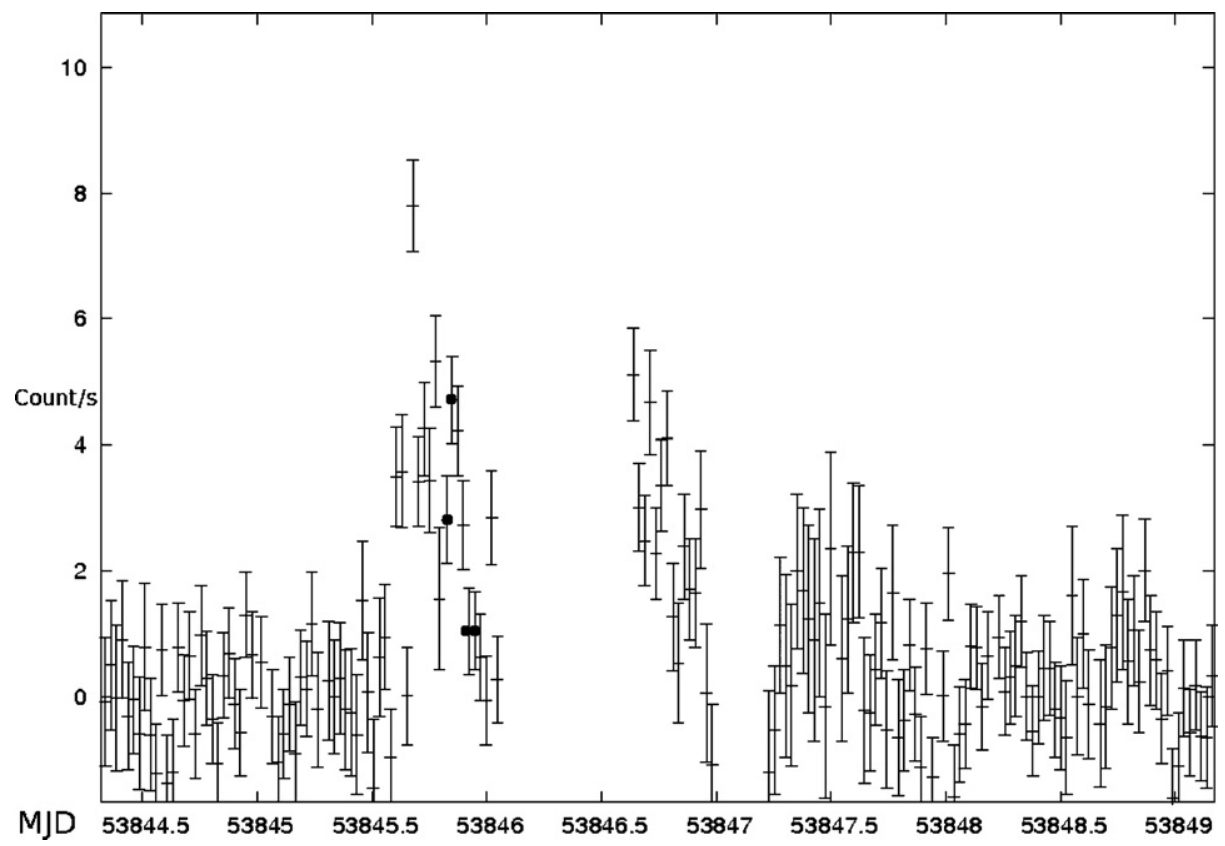

Figure 4. Zoomed view of the ISGRI light curve of flare D in Figure 3. Time and flux axes are in MJD and count s $\mathrm{s}^{-1}$, respectively. Each data point represents the average flux during one $\mathrm{ScW}(\sim 2000 \mathrm{~s})$. The black dots indicate the ScWs during which the source was also in the JEM-X FOV.

JEM-X data extend down only to $3 \mathrm{keV}$, i.e., not low enough in energy to fully allow an investigation of the absorption. We also performed the broadband spectral analysis of the simultaneous JEM-X/ISGRI flare spectrum: the best fit $\left(\chi_{v}^{2}=1.1,125\right.$ dof $)$ was achieved by an absorbed power law (see Figure 5) with $\Gamma=$ $2.5 \pm 0.6, N_{\mathrm{H}}=23_{-14}^{+19} \times 10^{22} \mathrm{~cm}^{-2}$ and a cross-calibration constant of $0.8_{-0.3}^{+0.8}$.

AX J1841.0-0536 was also detected at $\sim 5 \sigma$ level (4-20 keV) in the JEM-X1 mosaic (Figure 2 ) and at $\sim 4 \sigma$ level in the JEMX2 mosaic. With the aim of measuring its quiescent X-ray emission, we intentionally excluded from the JEM-X mosaic analysis those very few ScWs during which the source was detected in outburst. The 4-20 keV flux from both JEM-X1 and JEM-X2 detections is $\sim 2 \times 10^{-12} \mathrm{erg} \mathrm{cm}^{-2} \mathrm{~s}^{-1}$, such measurement likely represents the quiescent state of the source; indeed it is very similar to other values previously reported in the literature (Halpern et al. 2004; Sidoli et al. 2008). If we assume a distance of $\sim 6.9 \mathrm{kpc}$ then the corresponding X-ray luminosity is $\sim 1.1 \times 10^{34} \mathrm{erg} \mathrm{s}^{-1}$ and the dynamic range of the source is $\sim 10^{3}$. Unfortunately, the insufficient JEM-X statistics did not allow us to extract a meaningful spectrum and light curve of this quiescent state.

\subsubsection{Association with 3EG J1837-0423 or Alternatively with HESS J1841-055}

AX J1841.0-0536 is characterized by a striking spatial association with HESS J1841-055, however, its X-ray/soft gamma-ray behavior is completely different than that of the HESS source (pointlike and transient nature versus extended 


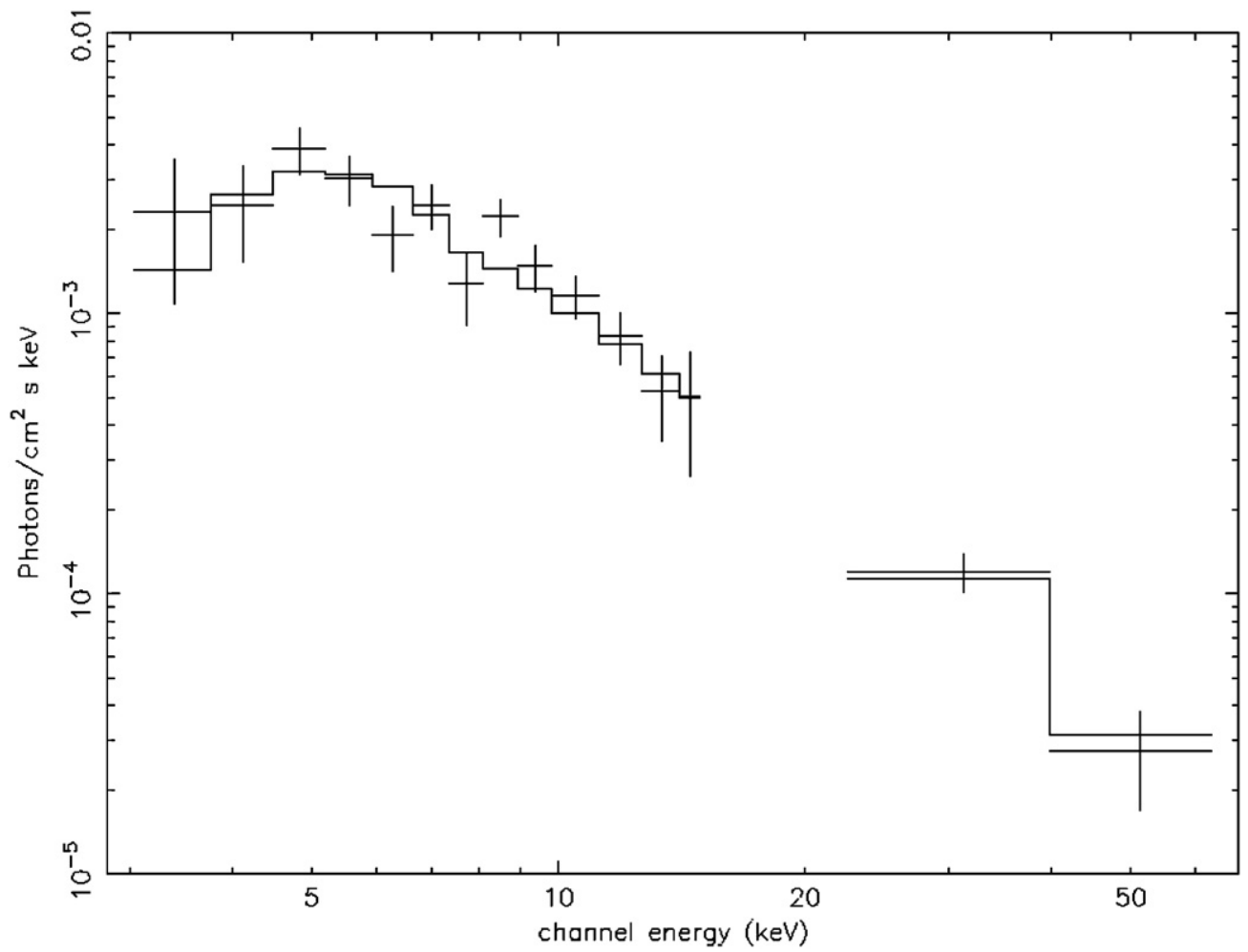

Figure 5. Unfolded combined JEM-X and ISGRI spectrum (3-60 keV) of AX J1841.0-0536 during flare D in Figures 3 and 4.

and nonvariable nature). Conversely, AX J1841.0-0536 and 3EG J1837-0423 share a similar fast transient behavior while their spatial match is not so precise. ${ }^{6}$ Despite these drawbacks, it is still worth studying the possible physical association of AX J1841.0-0536 with HESS J1841-055 or alternatively with 3EG J1837-0423.

In the case of HESS J1841-055, AX J1841.0-0536 is the only $\mathrm{X}$-ray (4-20 keV) and soft $\gamma$-ray (20-100 keV) object detected by INTEGRAL within the HESS error ellipse. It is located only $\sim 3^{\prime}$ from the HESS coordinates, i.e., right at the center of the $\mathrm{TeV}$ ellipse. Its transient $\mathrm{X}$-ray behavior as well as pointlike nature do not agree with the extended $\mathrm{TeV}$ emission seen in HESS J1841-055, however, it is still reasonable to postulate that AX J1841.0-0536 could be responsible for at least a fraction of the entire $\mathrm{TeV}$ emission. In principle, the three other cataloged objects located inside the HESS uncertainty region (the two radio pulsars PSR J1841-0524 and PSR J1838-0549 and the candidate SNR G26.6-0.1) could contribute to the remaining components of the $\mathrm{TeV}$ emission as it is largely known that PWN systems and SNRs are a prominent class of $\mathrm{TeV}$ galactic sources. In fact, $\sim 10 \mathrm{SNRs}$ have been detected at $\mathrm{TeV}$ energies and they show a clear extended $\mathrm{TeV}$ morphology. With its angular size of $\sim 10^{\prime}$, the candidate SNR G26.6-0.1 could possibly contribute to a component of the VHE emission from the extended TeV source HESS J1841-055. Although G26.60.1 was not detected at soft gamma-rays $(20-100 \mathrm{keV})$ by IBIS, this should not be taken as a strong proof element to exclude it as a possible counterpart because IBIS is not particularly suited for the detection of extended sources such as G26.6-0.1. As for PWN systems, they are generally associated with young and energetic pulsars; to date $\sim 20$ PWNs have been associated with pointlike $\mathrm{TeV}$ sources and almost all of them have also been

\footnotetext{
6 Positions of EGRET sources, being model background-dependent, should be considered only as indicative (Casandjian \& Grenier 2008).
}

detected at soft gamma-rays by IBIS. In our specific case, the two pulsars have not been detected by INTEGRAL in both energy bands 4-20 keV and 20-100 keV, despite very deep observations $(\sim 160 \mathrm{ks}$ and $\sim 3 \mathrm{Ms}$, respectively). Moreover, no cataloged PWN is associated with the pulsars and, if taken separately, each would require an impossible efficiency of $\sim 200 \%$ (Aharonian et al. 2008) to explain the $\mathrm{TeV}$ emission. All this casts doubts on the potential $\mathrm{TeV}$ nature of the two pulsars. Finally, it is very intriguing that a HMXB system with a neutron star and a supergiant companion (such as AX J1841.0-0536) is located right at the core of the TeV source HESS J1841-055, although we took into account the possibility that such an association could be simply a chance coincidence. To this aim we calculated the probability of finding a supergiant HMXB, such as AX J1841.0-0536, inside the HESS error ellipse by chance. Given the number of supergiant HMXBs detected by IBIS within the Galactic plane (Bird et al. 2007), defined here as restricted to a latitude range of $\pm 5^{\circ}$, we estimated a probability of $\sim 0.4 \%$, i.e., 0.15 chance coincidences are expected. Such a very low probability may suggest a real physical association between AX J1841.0-0536 and HESS J1841-055.

In the case of the transient 3EG J1837-0423, AX J1841.0 0536 is the only X-ray and soft $\gamma$-ray source detected in its immediate nearness and whose characteristics and behavior suggest a likely association. In fact, AX J1841.0-0536 spends the majority of the time in quiescence and very occasionally undergoes fast X-ray transient activity with a typical duration of a few hours, rarely a few days, i.e., similar to the transient activity of 3EG J1837-0423 recorded by EGRET. Therefore, we suggest that AX J1841.0-0536 is the best candidate counterpart to the peculiar and elusive $\gamma$-ray transient 3EG J1837-0423. As we previously reported in the case of HESS J1841-055, we calculated the probability of finding by chance a supergiant HMXB inside the 3EG J1837-0423 error circle with a radius 
of $\sim 1.2$. The estimated probability is equal to $\sim 5.4 \%$, such value is dominated by the considerably large chosen area (radius $\sim 1.2$ ); in fact if we consider the smaller area pertaining to the $95 \%$ probability contour (radius $\sim 0.7$ ) the chance probability drops to a lower value of $\sim 1.8 \%$. In summary, given the above numbers, we conclude that the association of AX J1841.0 0536 with 3EG J1837-0423 is possibly real although a chance coincidence cannot be excluded.

\section{ORIGIN OF MeV FLARES OR EXTENDED TeV EMISSION FROM AX J1841.0-0536}

Although the flaring behavior of both AX J1841.0-0536 and 3EG J1837-0423 along with the spatial proximity makes it tempting to postulate a physical relation, it remains open whether there is a physical mechanism to support the identification. The mechanisms proposed for the known $\mathrm{TeV}$ binaries and microquasars mentioned in Section 1 cannot be applied here, since in all those cases the sources are not X-ray transients. Systems like LS I +61 303, LS 5039, and PSR B1259-63 are not only persistent high-energy emitters, but also periodically variable sources, where the variability is modulated by the orbital period. Whatever produces the flares in AX J1841.0-0536 seems to have an intrinsically sporadic character.

It has been suggested that the fast flares of SFXTs like AX J1841.0-0536 are due to the interaction of the magnetized neutron star with clumps in the wind of the supergiant companion donor star (in't Zand 2005; Leyder et al. 2007; Walter \& Zurita Heras 2007; Negueruela et al. 2008). These clumps seem to be a common feature in the winds of hot stars (e.g., Owocki $\&$ Cohen 2006). The characteristics of the clumps are not well established, but densities of the order of $\rho \sim 10^{-12} \mathrm{~g} \mathrm{~cm}^{-3}$ and radii of $R_{\mathrm{cl}} \sim 10^{11} \mathrm{~cm}$ are likely (e.g., Romero et al. 2007b). Assuming a spherical shape, the mass of the clumps can be $M_{\mathrm{cl}} \sim 4 \times 10^{21} \mathrm{~g}$. The clumps will be accreted onto the neutron star only if the magnetospheric radius, $R_{\mathrm{M}}$, is less than the corotation radius, $R_{\Omega}$; otherwise centrifugal forces will expel the matter (Davidson \& Ostriker 1973; Stella et al. 1986):

$$
R_{\Omega}>R_{\mathrm{M}}
$$

The magnetospheric radius is obtained by balancing the matter pressure to the magnetic field pressure, i.e., $\rho V^{2}=B\left(R_{\mathrm{M}}^{2}\right) / 8 \pi$. Since $B\left(R_{\mathrm{M}}\right)=B_{\mathrm{NS}} R_{\mathrm{NS}}^{3} / R_{\mathrm{M}}^{3}$, this yields:

$$
\begin{aligned}
R_{\mathrm{M}}= & 2.6 \times 10^{6}\left(\frac{\rho_{\mathrm{cl}}}{\mathrm{g} \mathrm{cm}^{-3}}\right)^{-1 / 5}\left(\frac{B_{\mathrm{NS}}}{10^{12} \mathrm{G}}\right)^{2 / 5} \\
& \times\left(\frac{M_{\mathrm{NS}}}{M_{\odot}}\right)^{-1 / 5}\left(\frac{R_{\mathrm{NS}}}{10^{6} \mathrm{~cm}}\right)^{6 / 5} \mathrm{~cm},
\end{aligned}
$$

where $R_{\mathrm{NS}}$ is the radius of the neutron star, $M_{\mathrm{NS}}$ its mass, and $B_{\mathrm{NS}}$ its surface magnetic field. The subscript 'cl' refers to the clump. In estimating Equation (2) we considered that the infall velocity is $V=\left(2 G M_{\mathrm{NS}} / r\right)^{1 / 2}$ (e.g., Massi \& Kaufman Bernadó 2008).

The corotation radius is given by

$$
R_{\Omega}=\left(\frac{G M_{\mathrm{NS}} P^{2}}{4 \pi^{2}}\right)^{1 / 3}
$$

Here, $P=2 \pi / \Omega$ is the spin period. For a typical neutron star mass of $1.4 M_{\odot}$ and the observed period of $4.7 \mathrm{~s}$ for AX J1841.0-0536 (see Section 4.3.1) we obtain

$$
R_{\Omega}=4.7 \times 10^{8} \mathrm{~cm} .
$$

Fast X-ray flares with a peak luminosity of $L_{\mathrm{X}} \sim 10^{37} \mathrm{erg} \mathrm{s}^{-1}$ can be produced if the clump material impacts onto the surface of the neutron star. If a flare has a duration of the order of $\Delta t \sim 10^{4} \mathrm{~s}$, the accretion rate from the clump matter will be $\dot{M} \sim M_{\mathrm{cl}} / \Delta t \approx 4 \times 10^{17} \mathrm{~g} \mathrm{~s}^{-1}$. Around $10 \%$ of the rest mass energy of the accreted material is released as luminosity:

$$
L_{\mathrm{X}} \approx 0.1 \dot{M} c^{2} \mathrm{erg} \mathrm{s}^{-1} \sim 3.6 \times 10^{37} \mathrm{erg} \mathrm{s}^{-1} .
$$

This means that the sporadic interaction with massive clumps can explain the observed X-ray flares if the matter can reach the surface of the neutron star. However, Equation (2) imposes important constraints if we take into account the required energy budget. Since the infall velocity is determined by the mass of the neutron star which cannot depart too much from the canonical value of $1.4 M_{\odot}$, we are left with the sole possibility that the surface magnetic field of the pulsar should be $B_{\mathrm{NS}} \leqslant 1.8 \times 10^{12} \mathrm{G}$. Systems with long periods of $\sim 1000 \mathrm{~s}$ can accommodate even magnetars (Stella et al. 1986; Bozzo et al. 2008).

In the quiescent state, the X-ray luminosity of such system seems to be unusually high, $\sim 10^{34} \mathrm{erg} \mathrm{s}^{-1}$. The density contrast between the clumps and the background wind can reach values of $10^{3}-10^{5}$ (Runacres \& Owocki 2005). If we adopt the lower value, i.e., $\rho_{\mathrm{cl}} / \rho_{\text {wind }} \approx 10^{3}$, then the quiescent accretion rate will be $\dot{M} \sim M_{\text {wind }} / \Delta t \approx 4 \times 10^{14} \mathrm{~g} \mathrm{~s}^{-1}$. The quiescent X-ray luminosity results, therefore, $L_{\mathrm{X}} \sim 10^{34} \mathrm{erg} \mathrm{s}^{-1}$ in accordance with the observations (see Section 4.3.3). The requirement of $R_{\Omega}>R_{\mathrm{M}}$ then imposes $B_{\mathrm{NS}} \leqslant 1.6 \times 10^{10} \mathrm{G}$, according to Equation (2) and the value of $R_{\Omega}$. Then, in order to explain both the flaring and quiescent states as accretion onto a magnetized neutron star from a structured wind with clumps embedded in a background flow of low density, we need a low magnetic field in the star surface: $B_{\mathrm{NS}} \leqslant 10^{10} \mathrm{G}$.

Is there any room in this scenario for $\mathrm{MeV}-\mathrm{GeV} \gamma$-ray flares or extended TeV emission? Walter (2007) suggests that protons could be accelerated by multiple scattering of Alfvén waves in or close to the accretion column and then interact with material at the magnetospheric radius producing $\gamma$-rays through inelastic $p p$ collisions and the subsequent decays. He estimates maximum Lorentz factors for the protons of $\sim 10^{8}$, i.e., energies of $\sim 10^{17} \mathrm{eV}$. This suggestion is based on the work by Smith et al. (1992). In these calculations, only synchrotron proton losses are taken into account. However, the particle density in the acceleration region during the flares (the only occasion when the energetics is sufficient to sustain $\gamma$-ray luminosities of $10^{36} \mathrm{erg} \mathrm{s}^{-1}$ ) is huge, of the order of $\sim 10^{21} \mathrm{~cm}^{-3}$. In addition, the physical conditions in the polar column are rather extreme, with "temperatures" of $\sim 20 \mathrm{keV}$, as indicated by the ISGRI observations (Section 4.3.3), a photospheric emission area of $\sim 1 \mathrm{~km}^{2}$, and photon densities of $\sim 10^{24} \mathrm{~cm}^{-3}$ (Arons 1987). Under such conditions $p p$ and $p \gamma$ losses are catastrophic. The proton cooling timescale through inelastic collision with thermal protons is

$$
\left[t_{p}^{(p p)}\right]^{-1}=\frac{1}{E_{p}} \frac{d E_{p}}{d t}=n_{p} c \sigma_{p p} K^{(p p)},
$$

where $E_{p}$ is the proton energy, $n_{p}$ is the density of thermal protons, $K^{(p p)} \approx 0.5$ is the inelasticity (fraction of proton energy lost per interaction), and $\sigma_{p p} \sim 35 \mathrm{mb}$ is the $p p$ cross-section at $\mathrm{GeV}$ energies. Then, $t_{p}^{(p p)} \approx 2 \times 10^{-6} \mathrm{~s}$.

Synchrotron losses are given by

$$
\left[t_{p}^{\text {(synchr) }}\right]^{-1}=\frac{4}{3}\left(\frac{m_{e}}{m_{p}}\right)^{3} \frac{c \sigma_{\mathrm{T}} B^{2}}{8 \pi m_{e} c^{2}} \gamma_{p},
$$


where $\sigma_{\mathrm{T}}$ is the Thomson cross-section and the other symbols have their usual meaning. Then, for $B=10^{10} \mathrm{G}$, we have $t_{p}^{\text {(synchr) }}=0.6 \gamma_{p}^{-1} \mathrm{~s}$. This means that for protons of $1 \mathrm{TeV} p p$ losses are $3 \times 10^{2}$ times more important than synchrotron losses. The acceleration surely takes place at some distance from the surface of the pulsar, thus this is an absolute upper limit.

The acceleration rate of particles in the accretion column is (e.g., Begelman et al. 1990)

$$
\left[t_{p}^{(\mathrm{acc})}\right]^{-1}=\frac{\eta c e B}{E_{p}}
$$

where $\eta$ is the acceleration efficiency, which depends on the shock velocity and the mean free path of the particles. Typical velocities for turbulent motions in the accreting column are $\sim 10^{7} \mathrm{~cm} \mathrm{~s}^{-1}$ (Smith et al. 1992). Then, under the most favorable assumptions (diffusion in the Bohm limit), the efficiency is $\eta \sim 10^{-7}$. The maximum energy allowed for protons, taking into account the $p p$ losses, results in $E_{p}^{\max } \sim 1 \mathrm{GeV}$, in such a way that the protons are barely relativistic.

A most promising approach to generate relativistic particles during the accretion of the clump is the formation of a transient magnetic-tower jet that could carry away a fraction of the accreting material (Kato 2007). Magnetohydrodynamic simulations of the magnetic interaction between the neutron star field and the accreting material show that jets driven by magnetic pressure are formed along the rotation axis of the disk (Kato et al. 2004). This occurs when the accreting matter reaches distances of $\sim 40$ gravitational radii. In our case, this means $R_{\mathrm{M}}=8.4 \times 10^{6} \mathrm{~cm}$, which imposes an even more tight constraint onto the magnetic field: $B_{\mathrm{NS}} \sim 2.1 \times 10^{7} \mathrm{G}$. This value is well in accordance with the values expected in Atoll sources known to produce jets like Scorpius X-1 (Massi \& Kaufman Bernadó 2008). However, Atoll sources are low-mass X-ray binaries. Is it possible for a neutron star in an HMXB to have a magnetic field of $\sim 10^{7} \mathrm{G}$ ? We will briefly discuss the issue in what follows.

The accretion of matter onto neutron stars can produce strong temperature gradients that favor thermomagnetic processes and the decrease of the crustal conductivity, all this resulting in an accelerated magnetic field decay (e.g., Geppert \& Urpin 1994; Urpin \& Geppert 1995). The magnetic field in the crust of the neutron star is given by

$$
\frac{\partial \vec{B}}{\partial t}=-\frac{c^{2}}{4 \pi} \nabla \times\left(\frac{1}{\sigma} \nabla \times \vec{B}\right)+\nabla \times(\vec{u} \times \vec{B}) .
$$

Here,

$$
\frac{1}{\sigma}=\frac{1}{\sigma_{\mathrm{ph}}}+\frac{1}{\sigma_{\mathrm{imp}}}
$$

is the total conductivity in the crystallized crustal region, and $\vec{u} \propto \nabla T$ is the thermomagnetic velocity that characterizes the drift of the magnetic field under the influence of the temperature gradient. The conductivity is determined by the electrons, whose main scattering mechanisms are scattering on phonons and impurities (hence the terms $\sigma_{\mathrm{ph}}$ and $\sigma_{\mathrm{imp}}$, respectively). Since we are interested in the decay of a dipolar field, we can adopt a vector potential $\vec{A}=\left(0,, 0, A_{\varphi}\right)$, with $A_{\varphi}=s(r, t) \sin \theta / r$ (we are using spherical coordinates and standard notation). Since the drift is radially directed and the thickness of the crust is $\sim 0.1 R_{\mathrm{NS}}$, we can use the plane-parallel approximation (Geppert \& Urpin 1994) with the drift velocity in the $z$-direction (i.e., outward). Then, Equation (9) can be written as

$$
\frac{\partial s}{\partial t}=\frac{c^{2}}{4 \pi \sigma} \frac{\partial^{2} s}{\partial z^{2}}-u \frac{\partial s}{\partial z},
$$

with the condition $\partial s / \partial z=0$ at $z=0$. Given a prescription for the temperature, Equation (10) can be solved for different values of $\sigma_{\mathrm{imp}}$, since $\sigma_{\mathrm{ph}}$ is determined by the temperature and the crustal density $\left(\sim 10^{12} \mathrm{~g} \mathrm{~cm}^{-3}\right)$. The temperature profiles are determined by the accretion rate (Fujimoto et al. 1984). The accretion rate of dense clumps dominates the total accretion in a system like AX J1841.0-0536, as we have shown at the beginning of this section. Even with a very low duty cycle of $\sim 1 \%$, we have an average accretion rate of $\dot{M} \sim 10^{-10} M_{\odot}$ $\mathrm{yr}^{-1}$.

For a very pure crust, where the conductivity is basically determined by phonons, and the above-mentioned accretion rate, the thermomagnetic drift is directed outward and the crustal field expelled. The neutron star magnetic field then decays 4 orders of magnitude in $\sim 3 \times 10^{6} \mathrm{yr}$. At higher accretion rates or lower impurity content, the decay can be even larger in the same time. We conclude, then, that young neutron stars in HMXBs can, under certain conditions, have magnetic fields as low as those invoked in this paper (few times $10^{7} \mathrm{G}$ ). We note, however, that only the conductivity due to electron-phonon scattering depends on the temperature (which is sensitive to the accretion rate). The phonon conductivity decreases when $T$ increases, allowing changes in the magnetic field according to Equation (10). The conductivity due to electron-impurity scattering depends only on the number density of impurities in the crust. The value of $\sigma_{\text {imp }}$ is given by (e.g., Urpin \& Geppert 1995)

$$
\sigma_{\text {imp }} \sim 4.2 \times 10^{21} x \frac{Z}{Q} \mathrm{~s}^{-1} .
$$

In this expression, $x$ is a relativistic parameter of the electrons ( $x=p_{\mathrm{F}} / m c$, with $p_{\mathrm{F}}$ the Fermi pressure), $Z$ is the charge number of the dominant ions in the crust, and $Q$ is a parameter that characterizes the number density and charge of the impurities:

$$
Q=\frac{1}{n_{i}} \sum_{n^{\prime}} n^{\prime}\left(Z-Z^{\prime}\right)^{2},
$$

where $n_{i}$ is the number density of the dominant background ion species of charge number $Z$, and the primed parameters refer to the interloper species of impurities, over which the summation is carried out. Depending on the purity of the crust, the conductivity $\sigma_{\text {imp }}$ can dominate hindering the decrease of the magnetic field. This seems to be the case in accreting binaries with strong fields, like AO 0535+26 and similar systems.

The magnetic loops connecting the neutron star and the disk are twisted because of the differential rotation. Twist injection of matter from the disk results in the expansion of the loops, which creates a magnetic tower inside which the accelerated disk material is collimated as bipolar jets with subrelativistic speeds $(0.1-0.2 c)$. Magnetic reconnection at the base of the tower can inject plasmoids and the collision of plasmoids of different velocities will result in shocks in the outflow. Diffusive acceleration at these shocks can accelerate particles, both protons and electrons, up to relativistic energies. Electrons will cool almost instantly through synchrotron radiation producing X-rays (Romero \& Vila 2008). Actually, the conditions of the transient jet will not be very different from those discussed 
by these authors. Proton-photon interactions can produce $\gamma$ rays, secondary pairs, neutrinos, and neutrons according to the following reactions:

$$
p+\gamma \rightarrow p+a \pi^{0}+b\left(\pi^{+}+\pi^{-}\right)
$$

and

$$
p+\gamma \rightarrow n+\pi^{+}+a \pi^{0}+b\left(\pi^{+}+\pi^{-}\right),
$$

where $a$ and $b$ are the pion multiplicities. The decay chains for the mesons are

$$
\begin{gathered}
\pi^{+} \rightarrow \mu^{+}+v_{\mu}, \quad \mu^{+} \rightarrow e^{+}+v_{\mathrm{e}}+\bar{v}_{\mu}, \\
\pi^{-} \rightarrow \mu^{-}+\bar{v}_{\mu}, \quad \mu^{-} \rightarrow e^{-}+\bar{v}_{\mathrm{e}}+v_{\mu}, \\
\pi^{0} \rightarrow 2 \gamma .
\end{gathered}
$$

Proton-proton inelastic collisions would also yield $\gamma$-rays and secondary particles if the density of the ejected plasma is high enough:

$$
p+p \rightarrow p+\Delta^{+}+a \pi^{0}+b\left(\pi^{+}+\pi^{-}\right),
$$

where $\Delta^{+}$is a resonance that decays yielding a leading pion that takes around $17 \%$ of the proton energy.

The particle distribution for protons and those for all other types of particles (primary electrons, secondary pairs, muons, and pions) can be obtained solving the corresponding transport equations:

$$
\begin{aligned}
& \frac{\partial N_{i}(E, z, t)}{\partial t}+\frac{\partial}{\partial E}\left[\left.\frac{d E}{d t}\right|_{\text {loss }} N_{i}(E, z, t)\right]+\frac{N_{i}(E, z, t)}{t_{\mathrm{esc}}} \\
& +\frac{N_{i}(E, z, t)}{t_{\text {decay }}}=Q_{i}(E, t)
\end{aligned}
$$

where $t_{\mathrm{esc}}$ is the particle escape time from the acceleration region of thickness $\Delta z\left(t_{\text {esc }} \approx \Delta z / v_{\text {outflow }}\right), t_{\text {decay }}$ is the decay time for the different particles (infinity for $e$ and $p$ ), $d E /\left.d t\right|_{\text {loss }}$ is the sum of all losses for the type of particles considered, and $Q_{i}$ is the injection function that can be normalized in accordance to the energy budget of relativistic particles of type $i$ through

$$
L_{i}=\int_{V} d^{3} r \int_{E_{i}^{\min }}^{E_{i}^{\max }(z)} d E_{i} E_{i} Q_{i}\left(E_{i}\right)
$$

The injection, resulting from first-order Fermi acceleration mediated by the shocks formed in the magnetic tower, will have the form of a power law $Q_{p}\left(E_{i}\right) \propto E_{i}^{-\alpha}$, where $\alpha$ typically lies between 1.5 and 2.2 (Malkov \& O'C Drury 2001), depending of the shock geometry, nonlinear effects, etc. The particle distributions obtained solving Equation (19) will be affected by the losses. Reynoso \& Romero (2009) have solved Equation (19) in the steady state for protons, electrons, muons, and pions in conditions similar to those discussed in this paper. Romero \& Vila (2009) present the corresponding energy distributions due to all relevant processes: synchrotron radiation, IC scattering, relativistic Bremsstrahlung, $p \gamma$ and $p p$ interactions, and they include the effect of adiabatic losses as well. To solve the equations out of the steady state goes beyond the scope of the present paper. Nonetheless, some energetic considerations are in order to show that the proposed scenario is consistent.
We can assume, in accordance to the most recent studies of the accretion/ejection coupling (Körding et al. 2006), that around $10 \%$ of the accretion power is injected in the collimated outflow, and around $10 \%$ of this power is converted into power of relativistic particles, as it seems to be the case in microquasars and SNRs (e.g., Ginzburg \& Syrovatskii 1964; Bosch-Ramon et al. 2006). This means that we would have around $4 \times 10^{36} \mathrm{erg} \mathrm{s}^{-1}$ in relativistic particles injected during the accretion of the clump by the neutron star. So, a normal $\gamma$-ray flare would have around several times $10^{35} \mathrm{erg} \mathrm{s}^{-1}$ at energies $E_{\gamma}>100 \mathrm{MeV}$. These $\gamma$-rays would be the result of $p \gamma$ interactions close to the neutron star and $p p$ interactions in the dense medium around the system (the column density is $\sim 10^{23} \mathrm{~cm}^{-2}$ and the typical size of a massive binary system $\left.\sim 10^{12} \mathrm{~cm}\right)$. Protons could be confined by the local magnetic field. Secondary electrons and positrons from hadronic interactions can produce additional radiation through inverse Compton and synchrotron mechanisms (Orellana et al. 2007b; Bosch-Ramon et al. 2008). Very intense $\gamma$-ray flares such as those detected by EGRET can result from the accretion of a particularly large and dense clump. During the massive accretion period, when the source goes through the transient Atoll state, $\mathrm{X}$-ray pulsations are suppressed since the matter is directed outward. Pulsations are detectable during the quiescent states when the source accretes from the interclump medium.

Both $p \gamma$ and $p p$ produce neutrons, that cannot be confined by magnetic fields and could escape from the system. Depending on their energy these neutrons will decay at different distances, injecting both protons and electrons in the interstellar, dense medium around the binary system:

$$
n \rightarrow p+e^{-}+\overline{v_{e}} \text {. }
$$

The most energetic neutrons can reach distances of $d=$ $\gamma_{p}^{\max }(886.7 \mathrm{~s}) c \sim 1 \mathrm{pc}$. The protons from the decay of the neutrons will start to diffuse into the surrounding of the source, forming an extended, nonvariable, $\gamma$-ray source through the "illumination" in $\gamma$-rays of the ambient matter (e.g., BoschRamon et al. 2005). The total energy deposit in the medium by these neutrons during a flare will be $\sim 10^{36} \mathrm{erg} \mathrm{s}^{-1}$, which will be transferred to the decay products. If the duty cycle of the source is a few percent, then, on average, around $\sim 10^{34} \mathrm{erg} \mathrm{s}^{-1}$ will be injected. If particles have a spectral index of $\alpha \sim 2$, the most energetic particles will interact releasing around $10 \%$ of their energy in $\gamma$-rays up to pc-scale distances, sustaining an extended high-energy source. Note that the protons injected by the decay of neutrons have a long lifetime (see Equation (6), and consider an average molecular medium of $\sim 1-10 \mathrm{~cm}^{-3}$ ). The protons can be trapped by magnetic fields inside the binary system or the neighboring molecular clouds, being accumulated through many ejection episodes. Hence, an extended, stable source at high energies can co-exist with a transient X-ray and $\gamma$-ray compact source.

The above scenario is outlined only to show that the association of AX J1841.0-0536 with 3EG J1837-0423 or at least part of the emission from HESS J1841-055 is possible. A more detailed and general model, where SFXT sources are considered as low-magnetized pulsars undergoing sporadic changes to an Atoll-state, due to the accretion of clumps from the supergiant companion, will be presented elsewhere.

\section{DISCUSSIONS AND CONCLUSIONS}

We attempted to match, from an energetic and positional standpoint, the high-energy emission from 3EG J1837-0423 
and HESS J1841-055 with X-ray (4-20 keV) and soft $\gamma$-ray (20-100 keV) candidate counterparts detected through deep INTEGRAL observations.

In the case of HESS J1841-055, its TeV emission appears to have an extended morphology which suggests contributions from several sources; the possibility that the HESS source is the blend of more than one object has been explored. A search, using standard catalogs of sources which could be responsible of VHE emission, led to four cataloged objects positionally correlated with the HESS uncertainty region: two pulsars, a candidate SNR and an HMXB. The HMXB system AX J1841.0-0536 is the only object detected by INTEGRAL at X-ray $(4-20 \mathrm{keV})$ and at soft $\gamma$-rays (20-100 keV). Intriguingly, this HMXB is located at the core of the $\mathrm{TeV}$ emission. We estimated the probability of finding the supergiant HMXB AX J1841.0-0536 inside the HESS error ellipse by chance equal to $\sim 0.4 \%$, i.e., low enough not to preclude a physical association. We are aware that the pointlike nature and fast transient behavior of AX J1841.0 0536 exclude its association with the entire extended HESS source. Nevertheless, our study suggests that at least a fraction of the entire $\mathrm{TeV}$ emission might well be associated with $\mathrm{AX}$ J1841.0-0536. To this aim, we presented a theoretical model supporting such a scenario. It still remains to be understood what sources are then contributing to the rest of the $\mathrm{TeV}$ emission. It is largely known that SNRs are a prominent class of $\mathrm{TeV}$ galactic sources: as a consequence, the extended source and candidate SNR G26.6-0.1 (angular size $\sim 10^{\prime}$ ) could be responsible for a component of the entire $\mathrm{TeV}$ emission from HESS J1841055. PWN systems are also well-known TeV galactic sources, so in principle the two cataloged pulsars could be high-energy emitters which contribute to the entire $\mathrm{TeV}$ emission. However, there are the following doubts on their potential $\mathrm{TeV}$ nature: (1) no cataloged PWNs are associated with the two pulsars; (2) when taken separately, each pulsar would require an impossible high efficiency $(\sim 200 \%)$ to explain the VHE emission, not consistent with the range of efficiencies $(0.01 \%-11 \%)$ found for other TeV PWNs; (3) none of the two pulsars has been detected by INTEGRAL in the energy ranges 4-20 keV and 20$100 \mathrm{keV}$, despite very long observations, having in mind that almost all TeV PWN systems have been detected by INTEGRAL at soft gamma-rays. It is clear that the above information is not sufficient to draw any definitive conclusion on which other sources are contributing to the entire $\mathrm{TeV}$ emission from HESS J1841-055, apart from the HMXB AX J1841.0-0536. Further and deeper multiwavelength studies are strongly needed in order to (1) support or reject the $\mathrm{TeV}$ nature of the candidate SNR and the two pulsars and (2) unveil the presence of still undetected high-energy objects (i.e., PWNs, SNRs) inside the HESS error region.

As for the peculiar transient 3EG J1837-0423, to date no radio or X-ray counterpart has been found inside its $95 \%$ confidence error circle (radius $\sim 0.7 \mathrm{deg}$ ) despite extensive searches in the past years. Bearing this in mind, we opened the search for $\mathrm{X}$-ray and soft $\gamma$-ray counterparts to a bigger area, i.e., radius $\sim 1.2 \mathrm{deg}$. Among the few sources detected by INTEGRAL (4$20 \mathrm{keV}$ and $20-100 \mathrm{keV}$ ) in the proximity of 3EG J1837-0423, again we identified the transient HMXB AX J1841.0-0536 as the best candidate counterpart based on immediate nearness and similar flaring behavior. Furthermore, such association was supported from an energetic standpoint by proposing a theoretical mechanism able to explain the flaring $\mathrm{MeV}$ emission from AX J1841.0-0536. In the outlined scenario, AX J1841.0 0536 is a low magnetized pulsar $\left(B_{\mathrm{NS}} \sim 2.1 \times 10^{7} \mathrm{G}\right)$ which, due to the accretion of a massive clump from the supergiant companion, undergoes sporadic changes to a transient Atollstate where a magnetic tower can produce transient jets. After the collision with the massive clump, everything comes back to the normal state.

One way or another (association with HESS J1841-055 or alternatively with 3EG J1837-0423), the SFXT AXJ1841.0 0536 could be the prototype of a new class of Galactic transient $\mathrm{MeV} / \mathrm{TeV}$ emitters. Additional evidence for the existence of such a new class is also provided by very recent AGILE and Gamma-Ray Large Area Space Telescope (GLAST) discoveries on the Galactic plane of several unidentified transient $\mathrm{MeV}$ sources lasting only a few days (Tavani et al. 2008; Cheung et al. 2008; Pittori et al. 2008; Longo et al. 2008; Chen et al. 2007).

Further multiwavelength observations of the entire region in radio, X-rays (i.e., XMM, Chandra, and Swift/XRT), soft gamma-rays (i.e., INTEGRAL), $\mathrm{MeV}$ and $\mathrm{GeV}$ (i.e., AGILE and GLAST) are strongly needed in order to disentangle the emission possibilities, confirm or reject our proposed scenario, and find a definitive counterpart to the enigmatic and intriguing sources HESS J1841-055 and 3EG J1837-0423.

We thank the anonymous referee for useful comments which helped us to improve the quality of this paper. The Italian authors acknowledge the ASI financial support via grant ASI-INAF I/023/05/0, I/088/06/0/ and I/008/07/0/. G.E.R. thanks $\mathrm{V}$. Bosch-Ramon for insightful comments and the MPIfK, Heidelberg, for hospitality during his contribution to this work. $\mathrm{He}$ is supported by grants from CONICET and ANPCyT, as well as by the Ministerio de Educación y Ciencia (Spain) under grant AYA 2007-68034-C03-01, FEDER funds. This research has made use of data obtained from the HEASARC database.

\section{REFERENCES}

Aharonian, F., et al. 2005a, Science, 309, 746

Aharonian, F., et al. 2005b, A\&A, 442, 1

Aharonian, F., et al. 2008, A\&A, 477, 353

Albert, J., et al. 2006, Science, 312, 1771

Albert, J., et al. 2007, ApJ, 665, L51

Arons, J. 1987, in Proc. IAU Symp. 125, The Origin and Evolution of Neutron Stars, ed. D. J. Helfand \& J. H. Huang (Dordrecht: Reidel), 207

Bamba, A., et al. 2001, PASJ, 53, 1179

Begelman, M. C., Rudak, B., \& Sikora, M. 1990, ApJ, 362, 38

Bird, A. J., et al. 2007, ApJS, 170, 175

Bosch-Ramon, V., Aharonian, F. A., \& Paredes, J. M. 2005, A\&A, 432, 609

Bosch-Ramon, V., Khangulyan, D., \& Aharonian, F. A. 2008, A\&A, 482, 397

Bosch-Ramon, V., Romero, G. E., \& Paredes, J. M. 2006, A\&A, 447, 263

Bozzo, E., Falanga, M., \& Stella, L. 2008, ApJ, 683, 1031

Cardelli, J. A., Clayton, G. C., \& Mathis, J. S. 1989, ApJ, 345, 245

Casandjian, J.-M., \& Grenier, I. A. 2008, A\&A, 489, 849

Chen, A., et al. 2007, Astron. Telegr., 1308

Cheung, C. C., et al. 2008, Astron. Telegr., 1788

Davidson, K., \& Ostriker, J. P. 1973, ApJ, 179, 585

Dermer, C. D., \& Boettcher, M. 2006, ApJ, 643, 1081

Dubus, G. 2006, A\&A, 456, 801

Fujimoto, M. Y., Hanawa, T., Iben, I., Jr, \& Richardson, M. B. 1984, ApJ, 278, 813

Funk, S., et al. 2008, ApJ, 679, 1299

Geppert, U., \& Urpin, V. 1994, MNRAS, 271, 490

Ginzburg, V. L., \& Syrovatskii, S. I. 1964, The Origin of Cosmic Rays, (Oxford: Pergamon)

Halpern, J. P., Gotthelf, E. V., Helfand, D. J., Gezari, S., \& Wegner, G. A. 2004, Astron. Telegr., 289

Hartman, R. C., et al. 1999, ApJS, 123, 79

in't Zand, J. J. M. 2005, A\&A, 441, L1

Ishida, M., et al. 1998, A\&A, 336, 200

Kaspi, V. 2007, Ap\&SS, 308, 1 
Kato, Y. 2007, Ap\&SS, 307, 11

Kato, Y., Hayashi, M. R., \& Matsumoto, R. 2004, ApJ, 600, 338

Körding, E. G., Fender, R. P., \& Migliari, S. 2006, MNRAS, 369, 1451

Koyama, K., et al. 1989, PASJ, 41, 483

Koyama, K., et al. 1990, Nature, 343, 148

Kuiper, L., Hermsen, W., \& Mendez, M. 2004, ApJ, 613, 1173

Kuiper, L., et al. 2006, ApJ, 645, 556

Lang, K. R. 1992, Astrophysical Data: Planets and Stars (New York: Springer)

Leyder, J.-C., et al. 2007, A\&A, 465, L35

Liu, Q. Z., van Paradijs, J., \& van den Heuvel, E. P. J. 2007, A\&A, 469, 807

Longo, F., et al. 2008, Astron. Telegr. 1492

Lund, N., et al. 2003, A\&A, 411, L231

Malkov, M. A., \& O'C Drury, L. 2001, Rep. Prog. Phys., 64, 429

Maraschi, L., \& Treves, A. 1981, MNRAS, 194, 1

Massi, M., \& Kaufman Bernadó, M. 2008, A\&A, 477, 1

Misaki, K., et al. 1996, ApJ, 470, L53

Nagase, F. 1989, PASJ, 41, 1

Negueruela, I., et al. 2006, in Proc. the X-Ray Universe 2005 Vol. 1, ed. A. Wilson (Noordwijk: ESA), 165

Negueruela, I., et al. 2008, in AIP Conf. Proc. 1010, A Population Explosion, ed. R. M. Bandyopadhyay, S. Wachter, D. Gelino, \& C. R. Gelino (Melville, NY: AIP), 252

Nespoli, E., Fabregat, J., \& Mennickent, R. 2008, A\&A, 486, 911

Orellana, M., et al. 2007a, A\&A, 465, 703

Orellana, M., et al. 2007b, A\&A, 476, 9

Owocki, S. P., \& Cohen, D. H. 2006, ApJ, 648, 565

Paredes, J. M., Bosch-Ramon, V., \& Romero, G. E. 2006, A\&A, 451, 259

Pittori, C., et al. 2008, Astron. Telegr. 1394
Predehl, P., \& Schmitt, J. H. M. M. 1995, A\&A, 293, 889

Reynoso, M. M., \& Romero, G. E. 2009, A\&A, 493, 1

Rodriguez, J., et al. 2004, Astron. Telegr. 340

Romero, G. E., Christiansen, H. R., \& Orellana, M. 2005, ApJ, 632, 1093

Romero, G. E., Okazaki, A. T., Orellana, M., \& Owocki, S. P. 2007a, A\&A, 474,15

Romero, G. E., Torres, D. F., Kaufman Bernadó, M. M., \& Mirabel, I. F 2003, A\&A, 410, L1

Romero, G. E., \& Vila, G. S. 2008, A\&A, 485, 623

Romero, G. E., \& Vila, G. S. 2009, A\&A, 494L, 33

Romero, G. E., et al. 2007b, in Clumping in Hot Star Winds, ed. W.-R. Hamann, A. Feldmeier, \& L. Oskinova (Potsdam: Univ.-Verl.), 191

Runacres, M. C., \& Owocki, S. P. 2005, A\&A, 429, 323

Schlegel, D. J., Finkbeiner, D. P., \& Davis, M. 1998, ApJ, 500, 525

Sguera, V., et al. 2005, A\&A, 444, 221

Sguera, V., et al. 2006, ApJ, 646, 452

Sidoli, L., et al. 2008, ApJ, 687, 1230

Smith, I. A., Katz, J. I., \& Diamond, P. H. 1992, ApJ, 388, 148

Stella, L., White, N. E., \& Rosner, R. 1986, ApJ, 308, 669

Tavani, M., \& Arons, J. 1997, ApJ, 477, 439

Tavani, M., et al. 1997, ApJ, 479, L109

Tavani, M., et al. 2008, Astron. Telegr. 1827

Terada, Y., et al. 1999, PASJ, 51, 39

Terada, Y., et al. 2001, MNRAS, 328, 112

Thompson, D. J., et al. 1995, ApJS, 101, 259

Ubertini, P., et al. 2003, A\&A, 411, L131

Urpin, V., \& Geppert, U. 1995, MNRAS, 275, 1117

Walter, R. 2007, Ap\&SS, 309, 5

Walter, R., \& Zurita Heras, J. 2007, A\&A, 476, 335

Wegner, W. 1994, MNRAS, 270, 229 